Research Article

\title{
Curcumin-Loaded Mixed Micelles: Preparation, Characterization, and In Vitro Antitumor Activity
}

\author{
Suping Ji, ${ }^{1}$ Xiao Lin, ${ }^{1}$ Enjiang Yu, ${ }^{1}$ Chengyang Dian, ${ }^{1}$ Xiong Yan, ${ }^{1}$ Liangyao Li, ${ }^{1}$ \\ Meimei Zhang, ${ }^{3}$ Wenchang Zhao, ${ }^{1}$ and Linghui Dian $\mathbb{D}^{1,2}$ \\ ${ }^{1}$ School of Pharmaceutical Sciences, Guangdong Medical University, Dongguan 523808, China \\ ${ }^{2}$ Guangdong Key Laboratory for Research and Development of Natural Drugs, Guangdong Medical University, \\ Zhanjiang 524023, China \\ ${ }^{3}$ Affiliated Cancer Hospital and Institute of Guangzhou Medical University, Guangzhou 511436, China
}

Correspondence should be addressed to Linghui Dian; 605911308@qq.com

Received 17 October 2017; Accepted 25 December 2017; Published 15 March 2018

Academic Editor: Rachid Seqqat

Copyright $\odot 2018$ Suping Ji et al. This is an open access article distributed under the Creative Commons Attribution License, which permits unrestricted use, distribution, and reproduction in any medium, provided the original work is properly cited.

\begin{abstract}
The objective of this study was to prepare curcumin-loaded mixed Soluplus/TPGS micelles (Cur-TPGS-PMs) for oral administration. The Cur-TPGS-PMs showed a mean size of $65.54 \pm 2.57 \mathrm{~nm}$, drug encapsulation efficiency over $85 \%$, and drug loading of $8.17 \%$. The Cur-TPGS-PMs were found to be stable in various $\mathrm{pH}$ media ( $\mathrm{pH} 1.2$ for $2 \mathrm{~h}, \mathrm{pH} 6.8$ for $2 \mathrm{~h}$, and $\mathrm{pH} 7.4$ for $6 \mathrm{~h}$ ). The X-ray diffraction (XRD) patterns illustrated that curcumin was in the amorphous or molecular state within PMs. The In vitro release test indicated that Cur-TPGS-PMs possessed a significant sustained-release property. The cell viability in MCF-7 cells was found to be relatively lower in Cur-TPGS-PM-treated cells as compared to free Cur-treated cells. CLSM imaging revealed that mixed micelles were efficiently absorbed into the cytoplasm region of MCF-7 cells. Therefore, Cur-TPGS-PMs could have the significant value for the chronic breast cancer therapy.
\end{abstract}

\section{Introduction}

Curcumin (Cur), obtained from the rhizomes of Curcuma longa $\mathrm{L}$., turmeric, is the most widely used phytoconstituent in food industry. It has a wide spectrum of pharmacological activities, such as anti-inflammatory, anticancer, and other pharmacological activities [1]. Numerous experimental results had unequivocally demonstrated that curcumin could induce arrest and/or apoptosis of human cancer cells of a variety of solid tumors at, for example, colorectal, lung, breast, pancreatic, and prostate carcinomas [2-6]. Recently, a clinical trial performed in patients with familial adenomatous polyposis has confirmed that curcumin could decrease the progression of cancer at a variety of organ sites, showing the potentiality of chemoprevention [7]. Furthermore, considerable studies suggested that curcumin was an efficacious and safe compound for cancer therapy and chemoprevention. However, its limited aqueous solubility and degradation at alkaline $\mathrm{pH}$ hinder its bioavailability $[8,9]$. Following oral administration (up to $8 \mathrm{~g}$ per day), the compound is poorly absorbed with only trace appearing in the blood [10]. One of the attempts to improve its bioavailability is utilizing proper delivery vehicles to maximize the absorption of curcumin in the upper gastrointestinal tract. The nanodrug delivery system has a good prospect in oral administration, especially polymeric micelles. Polymeric micelles comprise inner and outer domains which are denominated "core" and "shell" respectively, which offer outstanding advantages to promote oral absorption of the chemotherapy drug through encapsulating with a hydrophobic drug in the cores [11, 12]. It can deliver the drug to the desired site at the concentration exceeding its intrinsic water solubility and protect the unstable drug from direct contacting with the gastrointestinal (GI) contents inducing chemical degradation and metabolism. Meanwhile, the encapsulated drug was sustained released, with direct uptake by cells. It should be emphasized that mixed micelles have received much attention due to increasing physical stability, improving drug-loading capacities, and enhancing 
antidilution ability compared with polymeric micelles for drug delivery [13]. Mixed micelles can self-assemble with two or more similar structures and performance block copolymers. Mixed micelles can provide multiple functionality micelles by constituent copolymers. Therefore, a mixed micelle was developed with Soluplus and D- $\alpha$-tocopheryl polyethylene glycol 1000 succinate (TPGS 1000) for curcumin delivery.

Soluplus is an amphiphilic polyvinyl caprolactampolyvinyl acetate-polyethylene glycol graft copolymer introduced by BASF recently. This graft copolymer has a polyethylene glycol (PEG) backbone as a hydrophilic part and vinylcaprolactam/vinyl acetate side chains as a hydrophobic structure. It can form micelles in aqueous solution above the CMC of $10^{-8} \mathrm{M}$ [14]. Though Soluplus could improve the oral bioavailability of poorly soluble drugs by producing solid solutions [15], so far there is no systemic research on preparation of drug-loaded Soluplus micelles.

D- $\alpha$-tocopheryl polyethylene glycol 1000 succinate (TPGS 1000), known as an attracting surfactant, has been used in many pharmaceutical nanoformulations, which can not only increase drug solubility and stability, enhance drug encapsulation efficiency, and prolong the time of drug retention in the blood but also improve the oral bioavailability of anticancer drugs and overcome the multidrug resistance by inhibiting P-gp efflux pumps [16]. Therefore, TPGS 1000-incorporating nanomicelle systems could successfully promote the oral absorption of anticancer drugs [17, 18].

The objective of this study was to develop a mixed micelle delivery system by using Soluplus and TPGS, which could solubilize curcumin in vitro to reach the clinically relevant concentration and deliver the drug in a controlled manner. Hence, we tried to develop Cur-TPGS-PMs to enhance its antitumor activity. After assessing the feasibility of preparation, we prepared and characterized the physicochemical properties of Cur-TPGS-PMs including particle size analysis, zeta potential measurement, drug entrapment efficiency, $\mathrm{pH}$ stability, and in vitro drug release behavior. Moreover, the toxicity and cell uptake of Cur-TPGS-PMs with MCF-7 breast cancer cells were further investigated comparing with free Cur. These results showed the promise of potential applications of delivering curcumin as a mixed micelle for cancer therapy.

\section{Materials and Methods}

2.1. Materials. Soluplus ${ }^{\circledR}$ (an amphiphilic polyvinyl caprolactampolyvinyl acetate-polyethylene glycol graft copolymer) was generously presented by BASF Auxiliary Chem. Co., Ltd. (Shanghai, China). D- $\alpha$-tocopherol polyethylene glycol 1000 succinate (TPGS 1000) was obtained from Eastman Chemical Company (USA). Curcumin (Cur) was provided by Sigma-Aldrich (USA). High-pressure liquid chromatography- (HPLC-) grade methanol was bought from Fisher Scientific (Beijing, China). Other chemical reagents received were used without further purification. Milli-Q grade water purified through a Millipore system (ELGA LabWater, Sartorius, UK) was used during this study.

2.2. Preparation of Micelles. Mixed micelles containing curcumin were prepared by an ameliorated film dispersion method [19]. Accordingly, curcumin and Soluplus were mixed into methanol. The mixed solution was evaporated under reduced pressure by a rotary evaporator at $35^{\circ} \mathrm{C}$, and then, a thin film with curcumin was achieved. The thin film was hydrated with deionized water containing TPGS, followed by moderate stirring for $2 \mathrm{~h}$. The curcumin-mixed Soluplus with TPGS-mixed micelles (abbreviated as Cur-TPGS-PMs) was obtained by filtration of the micelle through a $0.22 \mu \mathrm{m}$ polycarbonate membrane to separate nonencapsulated curcumin. In addition, curcumin Soluplus micelles (short form Cur-PMs) were prepared using the same protocol of curcumin-mixed Soluplus micelles except that TPGS were not used in the procedure, and used as the control.

2.2.1. Effect of TPGS Concentration. In order to select a suitable TPGS concentration for preparing the desirable Cur-TPGS-PMs, different concentrations (0.005\%, 0.015\%, $0.03 \%$, and $0.04 \%$ ) were investigated. Particle size and zeta potential were selected as the optimum condition for the Cur-TPGS-PM formulation.

2.2.2. Effect of Stirring Time. The stirring time for preparing the desirable Cur-TPGS-PMs was optimized. Particle size and zeta potential were selected as the optimum condition for the Cur-TPGS-PM formulation.

2.2.3. Effect of Stirring Rate. The stirring rate for preparing the desirable Cur-TPGS-PMs was investigated. Particle size and zeta potential were selected as the optimum condition for the Cur-TPGS-PM formulation.

2.2.4. Effect of Drug Loading. Cur-TPGS-PMs were prepared using different theoretical ratios of curcumin to polymer, that is, $0.5: 10,1: 10$, and $1.5: 10$, on the basis of the preliminary experiment to determine the optimum percentage of curcumin in the Soluplus matrix and its effects on particle size, polydispersity index (PDI), zeta potential, and encapsulation efficiency. The magnetic stirring time $(2 \mathrm{~h})$, stabilizer concentration ( $0.03 \%$ of TPGS), and aqueous ratio were kept constant.

2.3. Particle Size and Zeta Potential. Particle size is one of the important indexes in the evaluation of nanodosage forms. The average particle size and size distribution of Cur-TPGSPMs were determined by the Malvern Instruments Zetasizer Nano ZS90 (Malvern Instruments, Malvern, UK) laser particle size analyzer. Double dilution of the formulations with distilled water was analyzed for particle size and zeta potential before each measurement. The mean $\mathrm{Z}$-average diameter and polydispersity index (PDI) were obtained through cumulate analysis software. Each test was performed in triplicate.

2.4. Transmission Electron Microscopy (TEM). The morphological characteristics of Cur-TPGS-PMs were viewed by a transmission electron microscope (TEM, H66009IV, Hitachi, Japan). Cur-TPGS-PMs were diluted with distilled 
water and dropped on a copper grid covered with nitrocellulose. Samples were stained with oleic acid and dried at room temperature of $25^{\circ} \mathrm{C}$ before examination.

2.5. Characterization by X-Ray Diffraction (XRD). XRD analysis was carried out to determine the crystallinity of curcumin in PMs by an X-ray powder diffractometer (D-MAX 2200 VPC, Rigaku, Japan) operating with the voltage of $40 \mathrm{kV}$ and the current of $25 \mathrm{~mA}$. Data were collected in the $2 \theta$ range of 5-50 degrees at a scan rate of $0.9 \mathrm{~min}^{-1}$. A lyophilized powder sample was put on a glass plate to spread a flat surface for measurement. XRD patterns were obtained for free Cur, void PMs, physical mixture of void PMs and Cur, and Cur-TPGS-PMs.

2.6. pH-Dependent Stability Studies. The stability of CurTPGS-PMs was investigated under different $\mathrm{pH}(\mathrm{pH} 1.2, \mathrm{pH}$ 6.8 , and $\mathrm{pH} 7.4$ ) conditions that could impact their particle size and drug release properties. Briefly, $1 \mathrm{~mL}$ of Cur-TPGS$\mathrm{PM}$ colloidal solution was added into different $\mathrm{pH}$ solutions. The Cur-TPGS-PMs were incubated in $\mathrm{pH} 1.2$ for $2 \mathrm{~h}, \mathrm{pH} 6.8$ for $2 \mathrm{~h}$, and $\mathrm{pH} 7.4$ for $6 \mathrm{~h}$, respectively. The particle size and PDI were assessed after the incubation of Cur-TPGS-PMs with different $\mathrm{pH}$ solutions $[20,21]$.

2.7. In Vitro Release. The curcumin release from the CurTPGS-PM system was performed using a dialysis method. Dialysis bags (14000 MWCO, Millipore, Boston, MA, USA) were soaked in double-distilled water for $24 \mathrm{~h}$ before the experiment. Briefly, $1 \mathrm{~mL}$ of Cur-TPGS-PM colloidal solution, Cur-PM colloidal solution, or Cur suspension solution (equivalent to $1 \mathrm{mg}$ of Cur) was loaded into dialysis bags. The bags were in turn placed in a $150 \mathrm{~mL} \mathrm{pH} 1.2$ for $2 \mathrm{~h}$ and then in $\mathrm{pH} 7.4$ release media until $48 \mathrm{~h}$. The flask was placed in a water bath at $37 \pm 0.5^{\circ} \mathrm{C}$ under a shaking rate at $100 \mathrm{rpm}$. $5 \mathrm{~mL}$ of the release sample was withdrawn and replaced with the same volume of the fresh medium to maintain a sink condition at the specific time points of $0.5,1.0,1.5,2.0,4.0$, $8.0,12.0,24.0,36.0$, and $48.0 \mathrm{~h}$. The release samples were analyzed for curcumin content using a high-performance liquid chromatography (HPLC), and the release test on each sample was performed in triplicate.

The content of curcumin was assayed by an HPLC system (Shimadzu, Kyoto, Japan), which was equipped with an SPD-10A ultraviolet detector, LC-10AT pump. Curcumin separation was carried out on a column (Hypersil $\mathrm{C}_{18}$, $4.6 \times 250 \mathrm{~mm}, 5 \mu \mathrm{m}$, Yilite, China) with a mobile phase made up of methanol, water, and acetic acid $(76: 23: 1, \mathrm{v} / \mathrm{v} / \mathrm{v})$. The flow rate was maintained at $1 \mathrm{~mL} / \mathrm{min}$. The absorbance of curcumin was measured at a wavelength of $421 \mathrm{~nm}$.

2.8. Cell Culture. The MCF-7 cells (obtained from the American Type Culture Collection) were grown in an RPMI1640 medium with $10 \%(\mathrm{v} / \mathrm{v})$ heat-inactivated fetal bovine serum (FBS) and $1 \%$ antibiotics $(100 \mathrm{U} / \mathrm{ml}$ penicillin and $100 \mu \mathrm{g} / \mathrm{ml}$ streptomycin). The cells were maintained at $37^{\circ} \mathrm{C}$ with $5 \% \mathrm{CO}_{2}$ in a humidified incubator.
2.9. Cell Uptake Studies. To evaluate MCF-7 cellular uptake quantitatively, the MCF-7 cells were seeded into 6-well culture plates at a density of $3 \times 105$ cells/well under $5 \% \mathrm{CO}_{2}$ at $37^{\circ} \mathrm{C}$ for $24 \mathrm{~h}$. Free Cur, Cur-PMs, and Cur-TPGS-PMs were applied to 6-well culture plates for $4 \mathrm{~h}$. The final concentration of Cur was $10 \mu \mathrm{M}$. The culture medium was used as a blank control. The cells were washed 3 times with PBS (pH 7.4), fixed with $4 \%$ paraformaldehyde (v/v) for 15 min under $5 \% \mathrm{CO}_{2}$ at $37^{\circ} \mathrm{C}$, and trypsinized with $0.25 \%$ trypsinase and collected. The harvested cells were estimated with an FACScan flow cytometry to measure the fluorescence intensity of Cur for assessing the quantity of cellular uptake (excitation wavelength $=488 \mathrm{~nm}$, emission wavelength $=560 \mathrm{~nm}$ ).

MCF-7 cells were seeded into 6-well culture slides (BD Falcon) with densities being approximately $3 \times 10^{5}$ per well and allowed to culture for $24 \mathrm{~h}$. The cells were incubated with free Cur, Cur-PMs, and Cur-TPGS-PMs for $4 \mathrm{~h}$ at a final concentration of $20 \mu \mathrm{M}$ Cur. Then, the cells were washed with cold PBS (pH 7.4) three times to remove extracellular particles. The cell nuclei were stained with Hoechst $333342(5 \mathrm{mg} / \mathrm{mL})$ for $30 \mathrm{~min}$. Then, the cells were washed twice with PBS and observed with a laser scanning confocal microscope (Leica SP2, Heidelberg, Germany).

2.10. Cytotoxicity Assay. MCF-7 cells were seeded into 96-well culture plates at a density of $1 \times 10^{4}$ cells/well and cultured for $24 \mathrm{~h}$ under $5 \% \mathrm{CO}_{2}$ at $37^{\circ} \mathrm{C}$. When reaching approximately $80 \%$ confluence, the medium was then replaced with fresh culture media and different concentrations of free Cur, Cur-PMs, and Cur-TPGS-PMs for $48 \mathrm{~h}$, respectively. The final concentration of curcumin was in between 0 and $20 \mu \mathrm{M}$, and blank PMs were added as parallel controls. Blank culture medium was used as a blank control. After incubation for $48 \mathrm{~h}$, the cytotoxic effects were estimated by a sulforhodamine B (SRB, Sigma-Aldrich) staining assay [22]. Briefly, the culture medium was removed. The cells were fixed with trichloroacetic acid, washed with deionized water, and stained with SRB. The measuring absorbance was at $540 \mathrm{~nm}$ with a microplate reader (Model 680 microplate reader; Bio-Rad Laboratories, Hercules, CA, USA). The survivals of MCF-7 cells were evaluated using the following formula: survival $\%=\left(A_{540 \mathrm{~nm}}\right.$ for the treated cells $/ A_{540 \mathrm{~nm}}$ for the control cells) $\times 100 \%$, where $\mathrm{A}_{540 \mathrm{~nm}}$ denotes the absorbance at $540 \mathrm{~nm}$. Each test was performed in triplicate.

2.11. Statistical Analysis. Data are reported as the mean \pm SD (standard deviation). One-way analysis of variance was used to assess the significance among groups, after which post hoc tests with the Bonferroni correction were used for multiple comparisons between individuals. A value of $P$ less than 0.05 was evaluated to be statistically significant.

\section{Results}

3.1. Preparation of Mixed Micelles. Different process variables including stabilizer concentration, magnetic stirring time, magnetic stirring rate, and theoretical drug loading were optimized for the preparation of Cur-TPGS-PMs. 


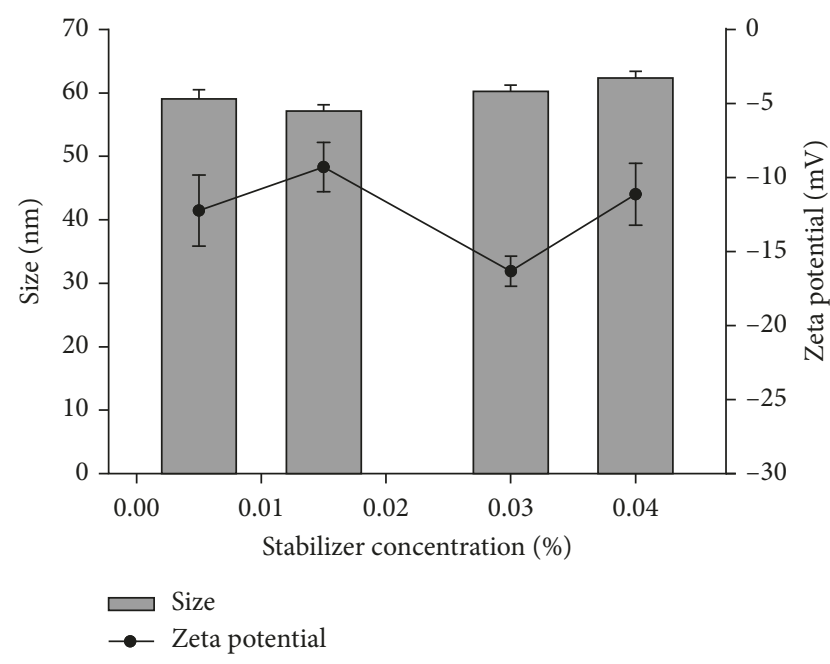

FIGURE 1: Effect of TPGS concentration on the size and zeta potential of Cur-TPGS-PMs.

3.1.1. Effect of Stabilizer Concentration. The effects of TPGS concentration on the particle size and zeta potential of CurTPGS-PMs are shown in Figure 1. As the concentration of TPGS increased (0.005-0.04\%), no significant difference was found in the particle size. The particle size was less than $100 \mathrm{~nm}$ which was acceptable. While the concentration of TPGS was $0.03 \%$, the absolute value of the zeta potential was the maximum, contributing to the stability of mixed micelles. Therefore, $0.03 \%$ TPGS (w/v) was optimized for the preparation of Cur-TPGS-PMs.

3.1.2. Effect of Stirring Time. Cur-TPGS-PMs were prepared by magnetic stirring for particle size reduction. Figure 2 shows the decrease in the particle size upon increasing the stirring time up to $2 \mathrm{~h}$. Magnetic stirring of $2 \mathrm{~h}$ brought about the smaller particle size $(59.32 \pm 1.01 \mathrm{~nm})$, and the absolute value of the zeta potential was the maximum. So, magnetic stirring of $2 \mathrm{~h}$ was selected for further research.

3.1.3. Effect of Stirring Rate. The effects of stirring rate on the particle size and zeta potential of Cur-TPGS-PMs were also studied. Figure 3 indicates that the effects of stirring rate on the particle size and zeta potential were negligible. When the stirring rate was $600 \mathrm{rpm}$, the zeta potential of Cur-TPGS-PMs reached the largest absolute value. So, the optimal magnetic stirring rate was set at $600 \mathrm{rpm}$ for further research.

3.1.4. Optimum of Drug Loading. The single film dispersion method was implemented for encapsulation of waterinsoluble curcumin in mixed micelles. Cur-TPGS-PMs were prepared with different theoretical ratios of curcumin to Soluplus, that is, $0.5: 10,1: 10$, and $1.5: 10$, to determine the optimum percentage of curcumin in nanocarriers. As shown in Table 1, the drug loading affected significantly the particle size and encapsulation efficiency of Cur-TPGS-PMs. With the increase of drug loading, the particle size increased and the encapsulation efficiency decreased significantly. The reason for

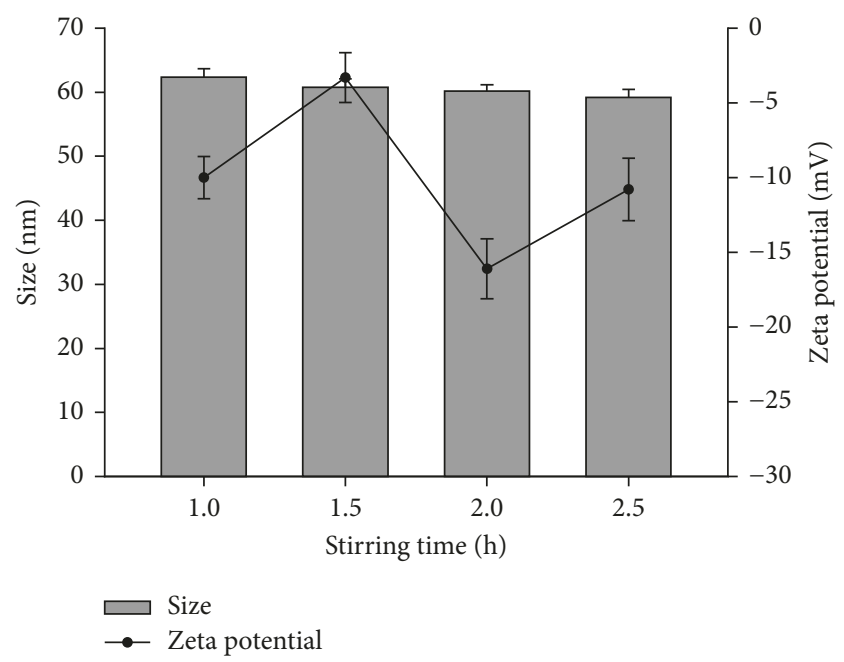

FIGURE 2: Effect of stirring time on the size and zeta potential of Cur-TPGS-PMs.

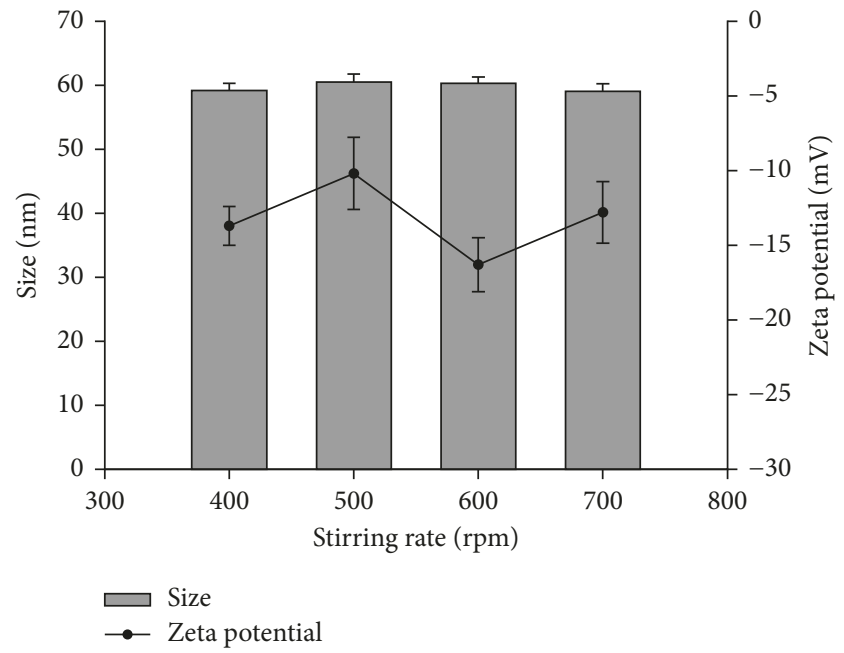

FIgURE 3: Effect of stirring rate on the size and zeta potential of Cur-TPGS-PMs.

that was more drug loading which expanded the core of micelles, leading to the increase of the particle size. Along with the improvement of ratios of drug to polymer, the encapsulation efficiency (EE) of Cur-TPGS-PMs declined markedly. It might be attributed to some amount of the polymer that could encapsulate a certain amount of drugs in mixed micelles. When the drug/Soluplus ratio was above $1.5: 10$, the micellar structure could break down. Therefore, the favorable drug loading in mixed micelle formulation was drug/Soluplus ratio at $1: 10$, in which the drug loading was superior and the CurTPGS-PMs had small particle size and uniform distribution.

\subsection{Characterization of Cur-TPGS-PMs}

3.2.1. Particle Size and Morphology. The average particle size of Cur-TPGS-PMs was $65.54 \pm 2.57 \mathrm{~nm}$ (Figure 4(a)), and 
TABLE 1: Effect of dosage on the size and zeta potential of Cur-TPGS-PMs (mean \pm SD, $n=3$ ).

\begin{tabular}{|c|c|c|c|c|}
\hline Curcumin: Soluplus & Particle size $(\mathrm{nm})$ & PDI & Zeta potential $(\mathrm{mV})$ & $\mathrm{EE}(\%)$ \\
\hline $0.5: 10$ & $60.76 \pm 1.02$ & $0.02 \pm 0.01$ & $-12.21 \pm 1.95$ & $93.17 \pm 0.92$ \\
\hline $1.0: 10$ & $67.13 \pm 2.01$ & $0.09 \pm 0.02$ & $-14.50 \pm 3.46$ & $90.26 \pm 1.38$ \\
\hline $1.5: 10$ & $506.41 \pm 5.02$ & $0.42 \pm 0.35$ & $-9.01 \pm 6.08$ & $51.34 \pm 2.94$ \\
\hline
\end{tabular}

Note. PDI, polydispersity index; SD, standard deviation; EE, encapsulation efficiency.

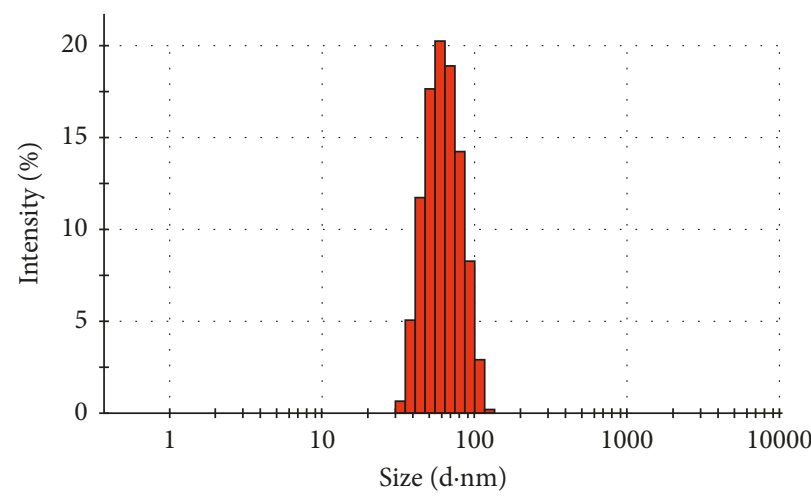

(a)

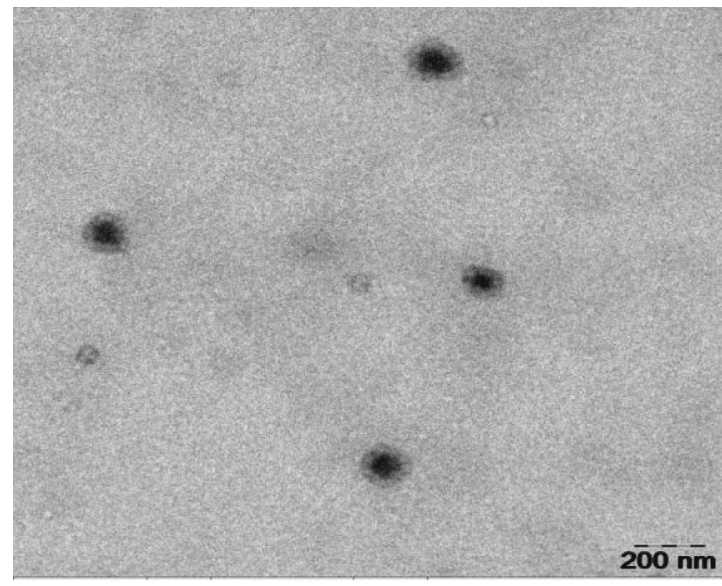

(c)

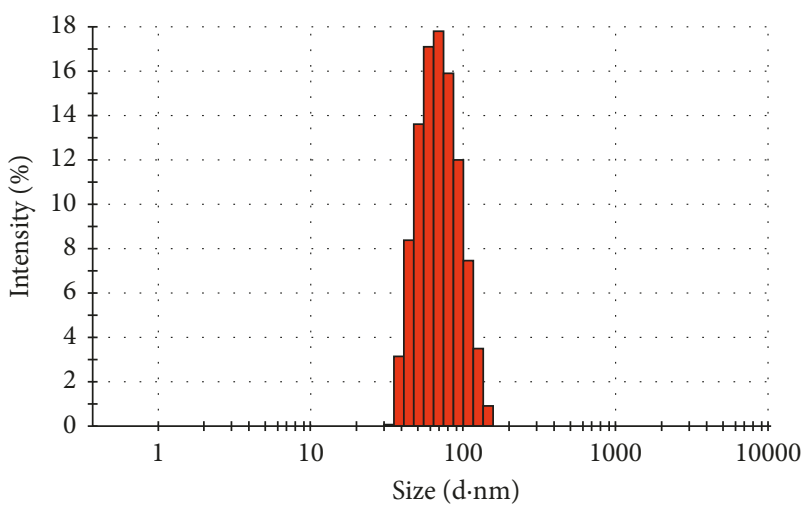

(b)

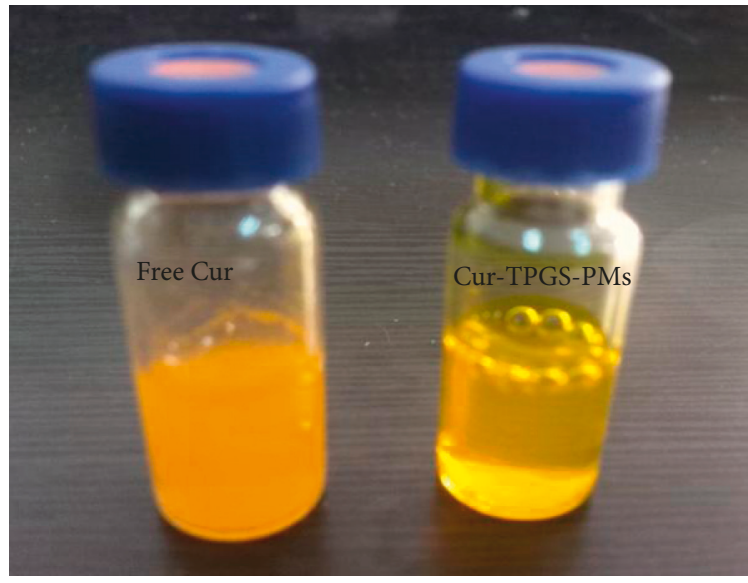

(d)

FIGURE 4: Particle size of blank-TPGS-PMs (a), particle size of Cur-TPGS-PMs (b), TEM image of Cur-TPGS-PMs (c), and colloidal solution of Cur-TPGS-PMs (right), in comparison with free Cur in water (left) (d).

the polydispersity index was $0.114 \pm 0.027$. Studies indicate that a favorable particle size range for oral absorption was advantageous to the proleptic tissue distribution and passive targeting ability of micelles in vivo $[23,24]$. It was well known that nanoparticles with the sizes ranging from $10 \mathrm{~nm}$ to $100 \mathrm{~nm}$ could show an optimum cellular and nuclear uptake in epithelial and smooth muscle cells [17]. Therefore, Cur-TPGS-PMs in small size were easier to be taken in intestines and could avoid rapid metabolism and elimination, with the stability and long circulation time guaranteed. Besides, the nanosized micelles were accounted for the enhanced permeability and retention (EPR) effect, that is, passive targeting ability. Thus, Cur-TPGS-PMs in small size with enhanced permeability and retention were suitable for tumor specific accumulation.
The average zeta potential measured was $-14.90 \pm 2.50 \mathrm{mV}$ with negative surface charge, which certainly increased the stability of Cur-TPGS-PMs in dispersion. The electrostatic repulsion and steric hindrance, as well as hydrophilic interactions between hydrophilic chains of the mixed micelle system, could prevent the aggregation of the micelles and provide important effects on stability of the colloid system [25].

The mixed micelles presented a perfectly monodispersed, spheroidal shape, and obvious core-shell structure with no conglutination under TEM (Figure 4(b)). With respect to the surface morphology, aqueous solution of Cur-TPGS-PMs was clear and transparent (Figure 4(c), right). In contrast, the equivalent amount of curcumin dispersed in water appeared turbid, nontransparent, and insoluble (Figure 4(c), left). Based on the amphiphilic property and block structure of 
carrier materials, it was deduced that the large hydrophobic chain area of Soluplus formed the core, and with the larger hydrophilic area, PEG distributed around the micelle core as an outer shell.

3.2.2. $X$-Ray Diffraction (XRD). XRD is a very useful technique to determine the status of the drug in carriers. The highly hydrophobic curcumin tends to crystallize from aqueous solution. If nanosized crystals are formed inside the matrix of Cur-TPGS-PMs, the drug elution from the PMs will be hindered. X-ray diffraction (XRD) analysis was performed to determine the physical status of curcumin encapsulated in Cur-TPGS-PMs as compared with that of free Cur. As shown in XRD patterns (Figure 5), free Cur appeared to be several crystal peaks that were characteristic of a crystalline structure in the $2 \theta$ range of $10-30^{\circ}$ [26]. The physical mixture of curcumin and void PMs also exhibited a lot of distinct peaks, suggesting that curcumin was crystalline in the mixture. No characteristic peaks of curcumin were seen in Cur-TPGSPMs, which hinted that the drug was molecularly dispersed or entrapped at an amorphous state in the micelles. In comparison with void PMs, the variation of position and spikes of the peaks in the XRD pattern of Cur-TPGS-PMs implied that minor interactions occurred between curcumin molecules and the polymer matrix during mixing.

3.2.3. pH-Dependent Stability Studies. Table 2 shows the stability of Cur-TPGS-PMs in different $\mathrm{pH}$. It was obvious that the particle size and PDI of Cur-TPGS-PMs had no significant change in $\mathrm{pH} 6.8$ or $\mathrm{pH} 7.4$ after incubation for $2 \mathrm{~h}$ or $6 \mathrm{~h}$. Although the particle size and PDI became larger (approximately $80 \mathrm{~nm}$ ) in $\mathrm{pH} 1.2$ after incubation for $2 \mathrm{~h}$, it was still in the best size range for oral absorption. Therefore, Cur-TPGS-PMs would prevent them from aggregation under various $\mathrm{pH}$ circumstances to promote their intestinal absorption.

3.2.4. In Vitro Release. To verify the potential of carriers to deliver the loaded drug, it is essential to assess the release profile of curcumin in vitro from the mixed micelle formulations. The in vitro release behaviors of tested formulations were observed in the $\mathrm{pH} 1.2$ for $2 \mathrm{~h}$ and then in the $\mathrm{pH} 7.4$ until $48 \mathrm{~h}$. As shown in Figure 6, the accumulative release of curcumin from free Cur, Cur-PMs, and Cur-TPGS-PMs in $\mathrm{pH} 1.2$ was about $3.21 \%, 1.06 \%$, and $1.61 \%$ for $2 \mathrm{~h}$, respectively. In the following release media ( $\mathrm{pH} 7.4)$, the release rates of free Cur were less than $20 \%$ after 48 h. From Figure 6, the releases of Cur-PMs and Cur-TPGS-PMs showed the sustainedrelease characteristics. The accumulative release rates of free Cur, Cur-PMs, and Cur-TPGS-PMs for $48 \mathrm{~h}$ were $19.64 \pm$ $1.65 \%, 24.91 \pm 2.62 \%$, and $32.11 \pm 2.22 \%$, respectively.

It could be concluded from abovementioned results that most of curcumin was embedded in the hydrophobic core by hydrophobic interaction or hydrogen bonds, and the curcumin in the surface of the particles was little [19]. The released mechanism of curcumin from micelles might be related to the drug diffusion and the disintegration of the

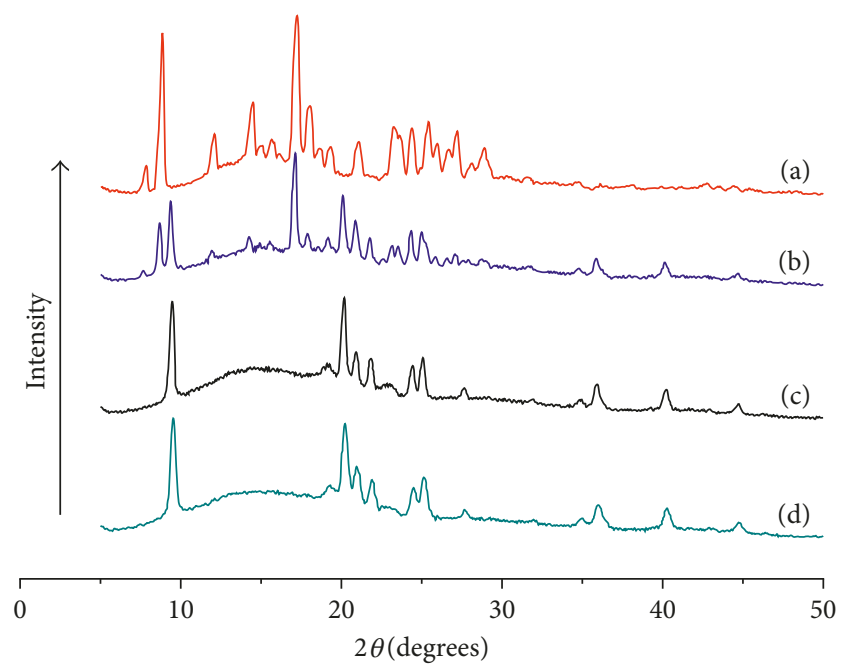

FIgURE 5: XRD patterns of free Cur (a), physical mixture of PMs and Cur (b), Cur-TPGS-PMs (c), and void PMs (d).

polymer material [27]. Curcumin was physically entrapped in the hydrophobic core of the mixed micelles that restricts its release from the micelles. Firstly, the media gradually got into the micellar interior to dissolve curcumin, and the dissolved drug spread to the media slowly. Then, the carrier material was slowly corroded and degraded, which resulted in the slow release of curcumin. Furthermore, the release of the drug from Cur-TPGS-PMs was faster than that from Cur-PMs. This is because the addition of TPGS in the formulation of Cur-TPGS-PMs increased the proportion of PEO, which was propitious to water molecules in the hydrophobic core of the mixed micelles, resulting in faster release of curcumin in Cur-TPGS-PMs.

In short, the fabricated formulation of Cur-TPGS-PMs possessed evidently a sustained-release profile, which may reduce the side effects of drugs, lessen the times of administration, decrease the fluctuations of blood drug concentration, and improve patient compliance.

3.3. Cellular Uptake. The fluorescence intensity of Cur was further quantitatively demonstrated by flow cytometry (Figure 2(b)). Results indicated that the mean fluorescence intensities after treatment with culture medium, free Cur, Cur-PMs, and Cur-TPGS-PMs for $4 \mathrm{~h}$ were $494 \pm 5.24,563.47 \pm 34.31$, $972.04 \pm 84.42$, and $1219.43 \pm 391.37$, respectively.

Cellular uptake was also determined by a confocal fluorescence microscope. The cell nucleus was stained with Hoechst 333342, and the micelles loaded with curcumin exhibited the green fluorescence. The various formulations were treated with MCF-7 cells for $4 \mathrm{~h}$. Figure 7 shows the confocal microscopic images of MCF-7 cells at $4 \mathrm{~h}$ after applying free Cur, Cur-PMs, and Cur-TPGS-PMs, respectively. Free Cur, Cur-PMs, and Cur-TPGS-PMs all showed definite uptake in the MCF-7 cells; however, the intensity of green fluorescence in the cell cytoplasm was evidently different. As shown in Figure 7, the highly hydrophobic free Cur was difficultly diffused into MCF-7 cells, leading to little cellular 
TABLE 2: Effect of $\mathrm{pH}$ on the particle size and PDI of Cur-TPGS-PMs (mean $\pm \mathrm{SD}, n=3$ ).

\begin{tabular}{lcccc}
\hline Medium & \multicolumn{2}{c}{ Initial } & & Final \\
& Particle size $(\mathrm{nm})$ & PDI & Particle size $(\mathrm{nm})$ & $79.48 \pm 2.64$ \\
pH 1.2 & $65.54 \pm 2.57$ & $0.11 \pm 0.04$ & $65.47 \pm 2.16$ & $0.22 \pm 0.03$ \\
pH 6.8 & $65.54 \pm 2.57$ & $0.11 \pm 0.04$ & $65.23 \pm 1.98$ & $0.11 \pm 0.05$ \\
pH 7.4 & $65.54 \pm 2.57$ & $0.11 \pm 0.04$ & $0.11 \pm 0.04$ \\
\hline
\end{tabular}

Note. PDI, polydispersity index; SD, standard deviation.

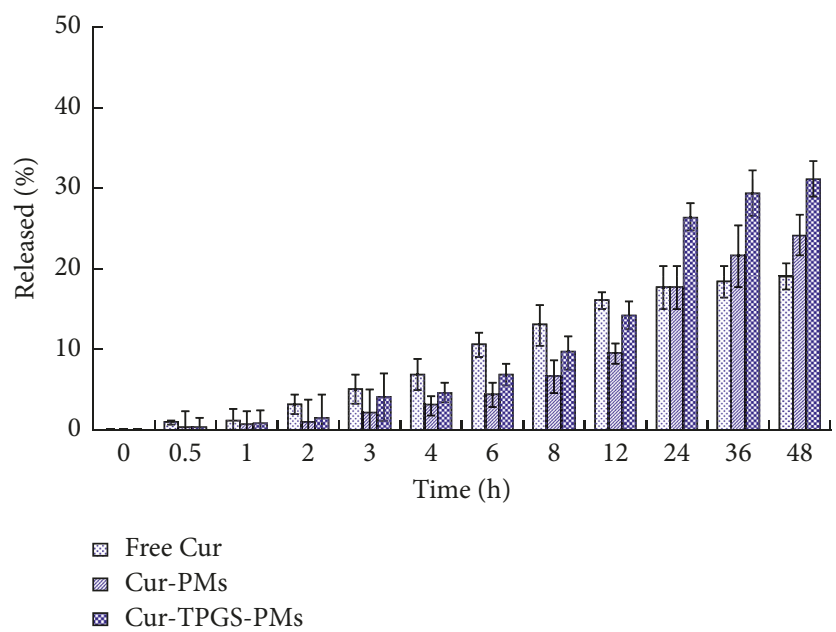

Figure 6: Curcumin release rates (\%) of free Cur, Cur-PMs, and Cur-TPGS-PMs in pH 1.2 for $2 \mathrm{~h}$ and $\mathrm{pH}$ 7.4.

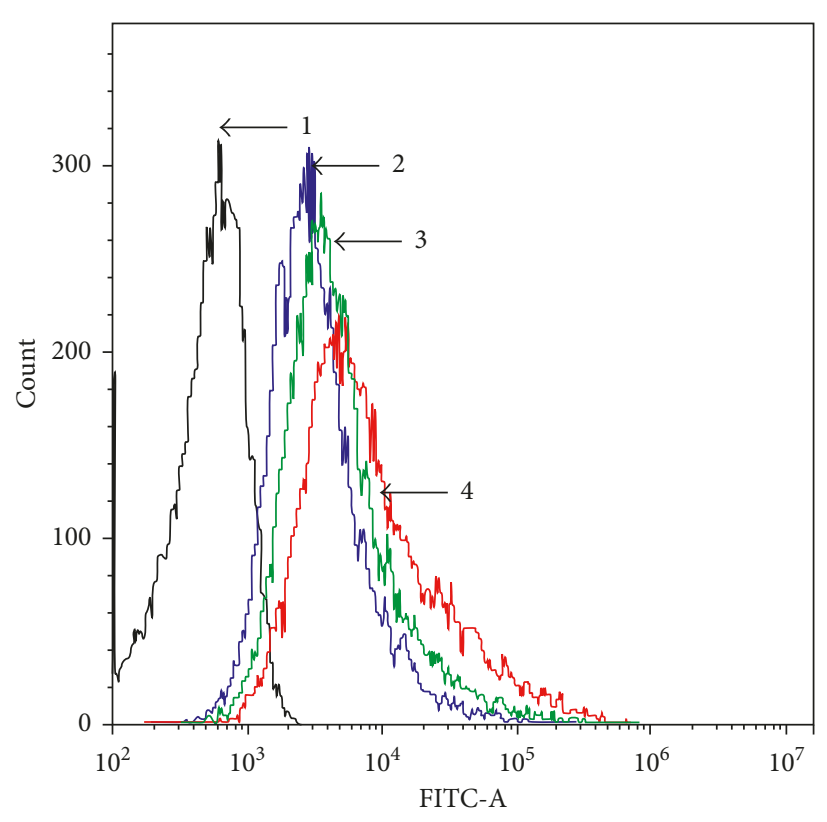

(a)

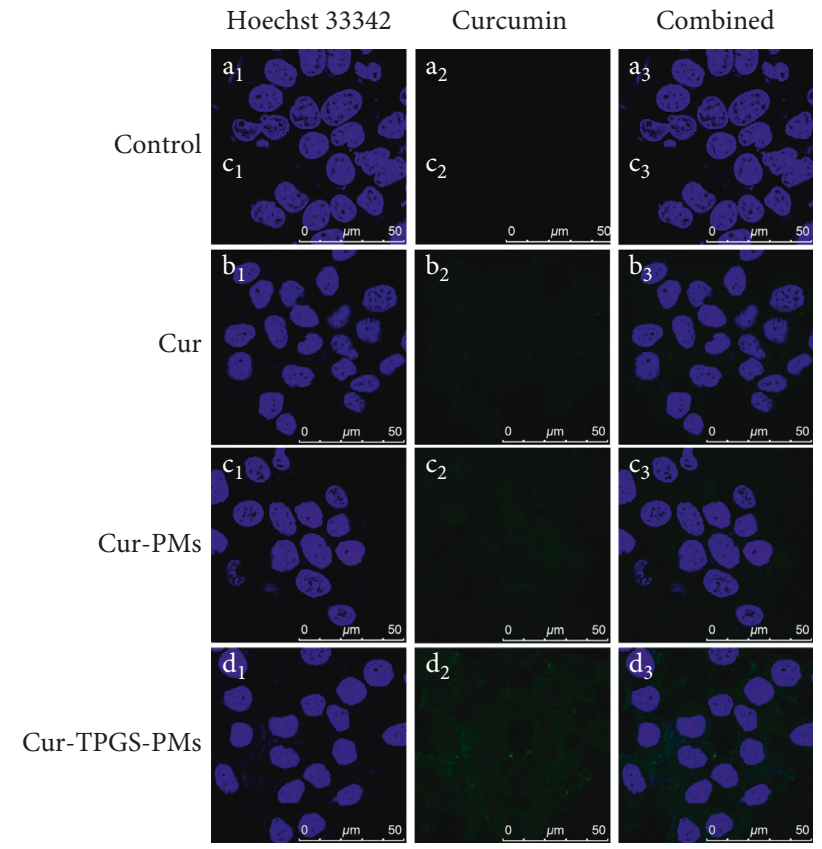

(b)

FIGURE 7: Intracellular uptake by MCF-7/Adr cells after treating with varying formulations by flow cytometry (a) and laser scanning confocal microscopy (b).

accumulation. The weak fluorescence in the free Cur-treated group could be attributed to the poor cellular internalization process because of only passive accumulation in the cells.
However, the overlaying images of Cur-TPGS-PMs in the cell cytoplasm displayed stronger green fluorescence of curcumin than that of free Cur and Cur-PM-treated cells. Here, TPGS 


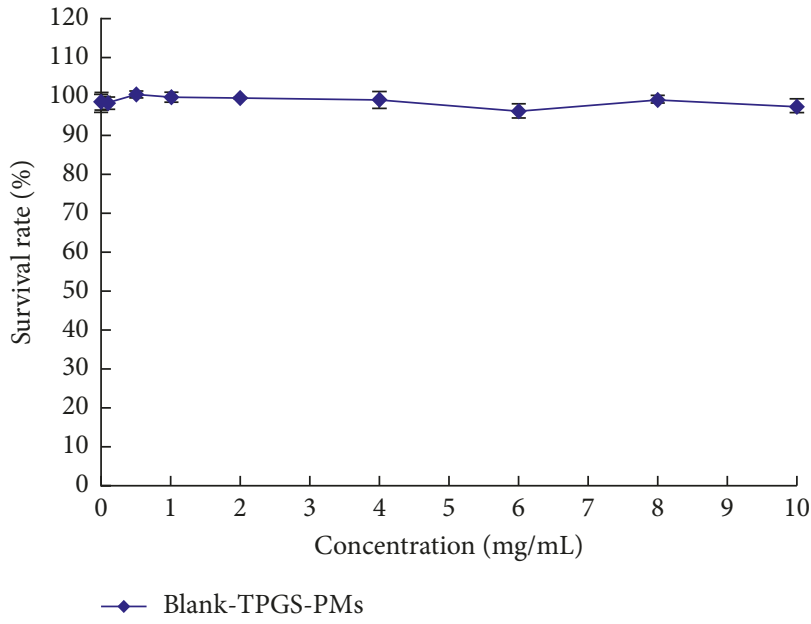

(a)

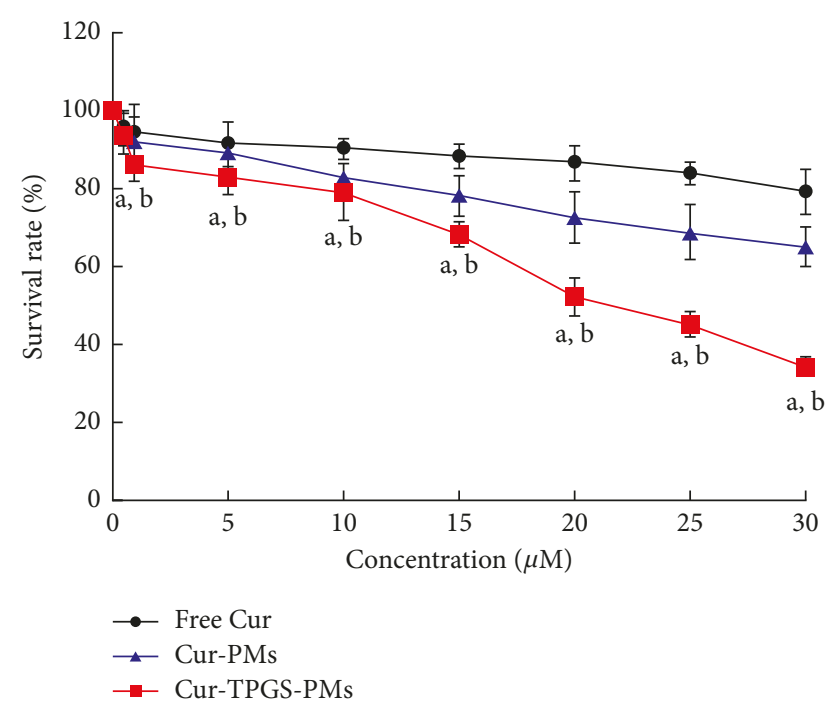

(b)

FIgURe 8: Survival rates of MCF-7 cells after treating with varying formulations. Data are presented as mean \pm SD $(n=3 ; P<0.05)$. (a) Blank-TPGS-PMs. (b) Free Cur; Cur-PMs; Cur-TPGS-PMs.

played an important part in improving the cellular uptake of curcumin. The cellular uptake of Cur-TPGS-PMs could be accelerated because they can be nonspecifically internalized into cells via endocytosis or phagocytosis [28]. Second, when Cur-TPGS-PMs attached to the cell membrane, the interactions between micelles and cell membranes can affect the structure and properties of lipid bilayers as well as functions of the biomacromolecules on the biomembrane, such as ion channels [29]. Therefore, the attachment of Cur-TPGS-PMs to the cell membrane is not as simple as physical adsorption. The homeostasis of cells can be consequently altered, which further contributed to the cytotoxicity of Cur-TPGS-PMs. Third, Cur-TPGS-PMs, possessing a markedly increased solubility and dissolution rate, could induce higher molecular concentration around the cells. The increased antitumor effect may also be associated with the more efficient uptake of tumor cells to the drug delivered.

3.4. Cellular Cytotoxicity. The cellular toxicity of free Cur, Cur-PMs, and Cur-TPGS-PMs was taken on MCF-7 breast cancer cells, which were sensitive and inhibited by curcumin [30]. Cell proliferation was assessed by SRB at the same concentration gradient $(0.01-20 \mu \mathrm{M})$. Figure 8 shows the cell survival rate after treatment with various concentrations of three kinds of formulations. The results indicated that free Cur, Cur-PMs, and Cur-TPGS-PMs showed a similar effect on cell viability after incubation for $48 \mathrm{~h}$. Free Cur did not show significant inhibition in MCF-7. The cell viability was relatively higher in free Cur-treated cells in comparison with Cur-TPGS-PM-treated cells. However, Cur-TPGS-PMs showed the highest inhibition rates on the proliferation of MCF-7 cells at various concentration points. The halfmaximal inhibitory concentration (IC 50 value) on MCF7 cells for free Cur, Cur-PMs, and Cur-TPGS-PMs was
$54.5,28.6$, and $10.3 \mu \mathrm{M}$, respectively. It was shown that IC 50 values of Cur-TPGS-PMs were the lowest among tested formulations. Meanwhile, the empty micelles, just containing TPGS and Soluplus, showed negligible effect on MCF-7 cell survival, which indicated that carrier materials were safe (Figure 8). In short, the results suggested that Cur-TPGS-PMs significantly improved the antitumor efficacy of curcumin.

\section{Conclusions}

In this study, curcumin was loaded within Soluplus-based mixed micelles using a modified film dispersion method. The Cur-TPGS-PMs with narrow size distribution had good entrapment efficiency, sustained-release character, and excellent long-term physical stability. The Cur-TPGS-PMs provide a promising preparation with efficient delivery of curcumin for therapeutic treatment in the near future. The anticancer activity of curcumin has been an extensive research, and it has been indicated as a potential agent for both prevention and treatment of a great variety of different cancers, including breast cancer, lung cancer, and sarcoma [3]. Moreover, this study explored an interesting alternative approach for design and fabrication of mixed micelles as delivery systems for curcumin.

\section{Disclosure}

The authors alone are responsible for the content and writing of this article.

\section{Conflicts of Interest}

The authors report no conflicts of interest. 


\section{Acknowledgments}

The authors gratefully acknowledge Science and Technology Planning Project of Guangdong Province (2014A020212296), Administration of Traditional Chinese Medicine of Guangdong Province, China (20161140, 20151258), Medical Scientific Research Foundation of Guangdong Province, China (A2016076, A2017106), Social Development Key Project of Dongguan (2017507152463), Key cultivation project of Guangdong Medical University (Z2016002), and National Natural Science Foundation of China (81473401) for the financial support. The study was supported in part by the Central Finance Fund to Develop Local Universities (Construction of Tumor Nano-Pharmaceutics Innovative Team, 2016).

\section{References}

[1] R. C. Srimal, "Turmeric: a brief review of medicinal properties," Fitoterapia, vol. 68, pp. 483-493, 1997.

[2] R. K. Maheshwari, A. K. Singh, J. Gaddipati, and R. C. Srimal, "Multiple biological activities of curcumin: a short review," Life Sciences, vol. 78, no. 18, pp. 2081-2087, 2006.

[3] A. Duvoix, R. Blasius, S. Delhalle et al., "Chemopreventive and therapeutic effects of curcumin," Cancer Letters, vol. 223, no. 2, pp. 181-190, 2005.

[4] B. B. Aggarwal, A. Kumar, and A. C. Bharti, "Anticancer potential of curcumin: preclinical and clinical studies," Anticancer Research, vol. 23, no. 1, pp. 363-398, 2003.

[5] Z. Wang, Y. Zhang, S. Banerjee et al., "Notch-1 downregulation by curcumin is associated with the inhibition of cell growth and the induction of apoptosis in pancreatic cancer cells," Cancer, vol. 106, no. 11, pp. 2503-2513, 2006.

[6] S. L. Ari, L. Strier, D. Kazanov et al., "Celecoxib and curcumin synergistically inhibit the growth of colorectal cancer cells," Clinical Cancer Research, vol. 11, no. 18, pp. 6738-6744, 2005.

[7] C. M. Correa, D. A. Shoskes, P. Sanchez et al., "Combination treatment with curcumin and quercetin of adenomas in familial adenomatous polyposis," Clinical Gastroenterology and Hepatology, vol. 4, no. 8, pp. 1035-1038, 2006.

[8] H. H. Tonnesen and J. Karlsen, "Studies on curcumin and curcuminoids," Zeitschrift für Lebensmittel-Untersuchung und Forschung, vol. 180, no. 5, pp. 402-404, 1985.

[9] Y. J. Wang, M. H. Pan, A. L. Cheng et al., "Stability of curcumin in buffer solutions and characterization of its degradation products," Journal of Pharmaceutical and Biomedical Analysis, vol. 15, no. 12, pp. 1867-1876, 1997.

[10] A. L. Cheng, J. K. Lin, M. M. Hsu et al., "Phase I chemoprevention clinical trial of curcumin, a chemopreventive agent, in patients with high risk or pre-malignant lesions," Anticancer Research, vol. 21, no. 4, pp. 2895-2900, 2001.

[11] K. Kataoka, A. Harada, and Y. Nagasaki, "Block copolymer micelles for drug delivery: design, characterization and biological significance," Advanced Drug Delivery Reviews, vol. 64, pp. 37-48, 2012.

[12] L. Bromberg, "Polymeric micelles in oral chemotherapy," Journal of Controlled Release, vol. 128, no. 2, pp. 89-112, 2008.

[13] A. B. E. Attia, Z. Y. Ong, J. L. Hedrick et al., "Mixed micelles self-assembled from block copolymers for drug delivery," Current Opinion in Colloid and Interface Science, vol. 16, no. 3 , pp. 182-194, 2011.

[14] S. Ali, N. Langley, D. Djuric, and K. Kolter, "Eye on excipients," https://industries.basf.com/bin/bws/documentDownload. en. 8805804190165 .
[15] R. N. Shamma and M. Basha, "Soluplus ${ }^{\circledR}$ : a novel polymeric solubilizer for optimization of carvedilol solid dispersions: formulation design and effect of method of preparation," Powder Technology, vol. 237, pp. 406-414, 2013.

[16] Y. Y. Guo, J. Luo, S. W. Tan et al., "The applications of Vitamin E TPGS in drug delivery," European Journal of Pharmaceutical Sciences, vol. 49, no. 2, pp. 175-186, 2013.

[17] S. S. Feng, L. Mei, P. Anitha, C. W. Gan, and W. Zhou, "Poly (lactide)-vitamin E derivative/montmorillonite nanoparticle formulations for the oral delivery of Docetaxel," Biomaterials, vol. 30, no. 19, pp. 3297-3306, 2009.

[18] M. J. Shieh, C. Y. Hsu, L. Y. Huang, H.-Y. Chen, F.-H. Huang, and P.-S. Lai, "Reversal of doxorubicin-resistance by multifunctional nanoparticles in MCF-7/ADR cells," Journal of Controlled Release, vol. 152, no. 3, pp. 418-425, 2011.

[19] L. H. Dian, E. J. Yu, X. N. Chen et al., "Enhancing oral bioavailability of quercetin using novel soluplus polymeric micelles," Nanoscale Research Letters, vol. 9, no. 1, pp. 648-659, 2014.

[20] D. R. Kalaria, G. Sharma, V. Beniwal, and M. N. V. Ravi Kumar, "Design of biodegradable nanoparticles for oral delivery of doxorubicin: in vivo pharmacokinetics and toxicity studies in rats," Pharmaceutical Research, vol. 26, no. 3, pp. 492-501, 2009.

[21] E. Zimmermann and R. H. Müller, "Electrolyte- and pHstabilities of aqueous solid lipid nanoparticle (SLN) dispersions in artificial gastrointestinal media," European Journal of Pharmaceutics and Biopharmaceutics, vol. 52, no. 2, pp. 203210, 2001.

[22] X. Y. Li, Y. Zhao, M. G. Sun et al., "Multifunctional liposomes loaded with paclitaxel and artemether for treatment of invasive brain glioma," Biomaterials, vol. 35, no. 21, pp. 5591-5604, 2014.

[23] H. Cabral, Y. Matsumoto, K. Mizuno et al., "Accumulation of sub-100 nm polymeric micelles in poorly permeable tumours depends on size," Nature Nanotechnology, vol. 6, no. 12, pp. 815-823, 2011.

[24] Z. Popovic, W. Liu, V. P. Chauhan et al., "A nanoparticle size series for in vivo fluorescence imaging," Angewandte Chemie International Edition, vol. 49, no. 46, pp. 8649-8652, 2010.

[25] M. Larsson, A. Hill, and J. Duffy, "Suspension stability: why particle size, zeta potential and rheology are important," Annual Transactions of the Nordc Rheology Society, vol. 20, pp. 209-214, 2012.

[26] K. Hu, X. Huang, Y. Gao, X. Huang, H. Xiao, and D. J. McClements, "Core-shell biopolymer nanoparticle delivery systems: synthesis and characterization of curcumin fortified zein-pectin nanoparticles," Food Chemistry, vol. 182, pp. 275-281, 2015.

[27] L. C. Chen, Y. C. Chen, C. Y. Su, W.-P. Wong, M.-T. Sheu, and H.-O. Ho, "Development and characterization of lecithinbased self-assembling mixed polymeric micellar (saMPMs) drug delivery systems for curcumin," Scientific Reports, vol. 6, no. 1, pp. 37122-37133, 2016.

[28] H. Hillaireau and P. Couvreur, "Nanocarriers' entry into the cell: relevance to drug delivery," Cellular and Molecular Life Sciences, vol. 66, no. 17, pp. 2873-2896, 2009.

[29] Z. Sezgin, N. Yuksel, and T. Baykara, "Investigation of pluronic and PEG-PE micelles as carriers of meso-tetraphenyl porphine for oral administration," International Journal of Pharmaceutics, vol. 332, no. 1-2, pp. 161-167, 2007.

[30] F. S. T. Mirakabad, A. Akbarzadeh, and M. Milani, "A comparison between the cytotoxic effects of free curcumin and curcumin-loaded PLGA-PEG nanoparticles on the MCF7 human breast cancer cell line," Artificial Cells, Nanomedicine, and Biotechnology, vol. 44, no. 1, pp. 423-430, 2014. 


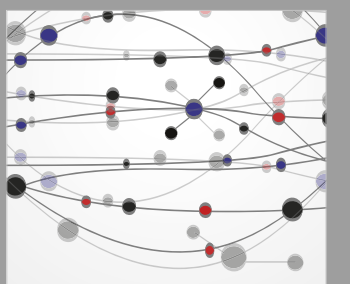

The Scientific World Journal
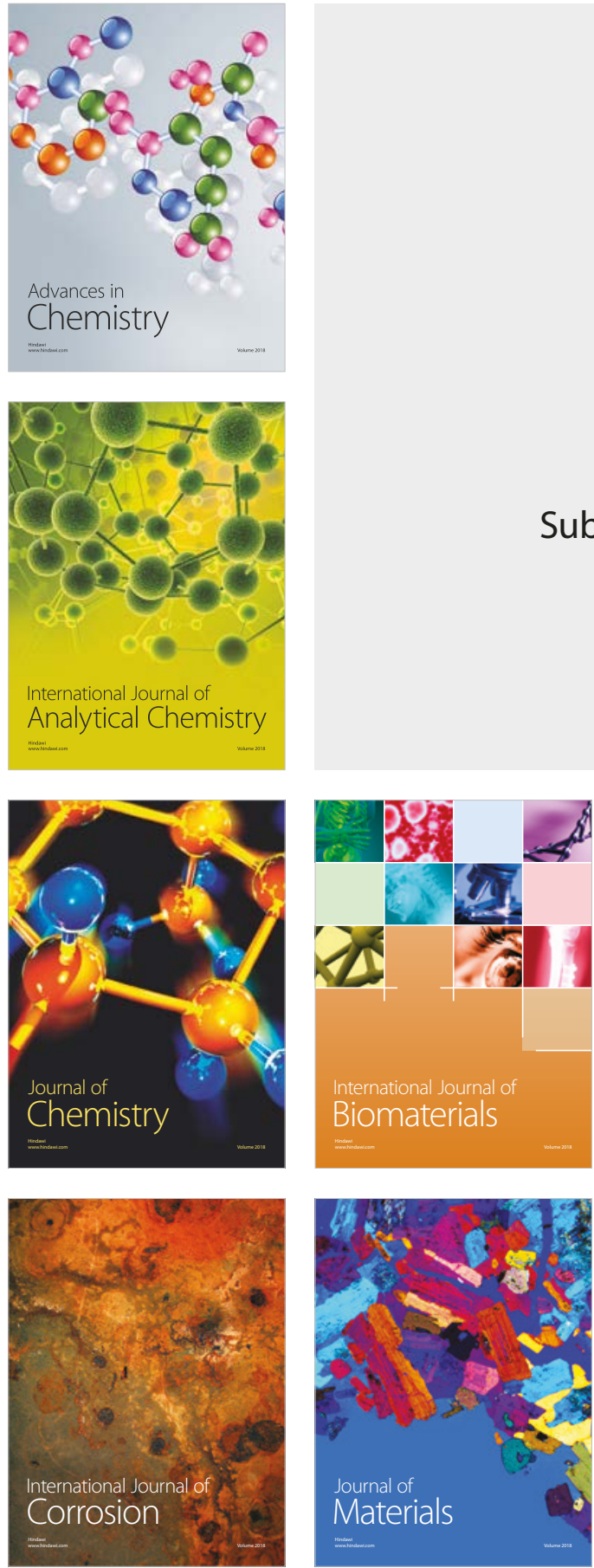

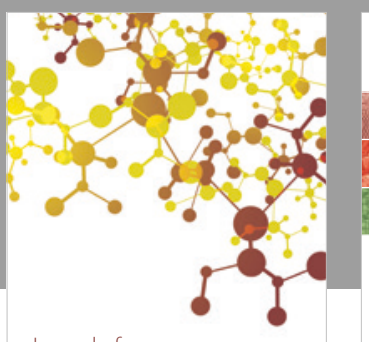

Journal of

Applied Chemistry
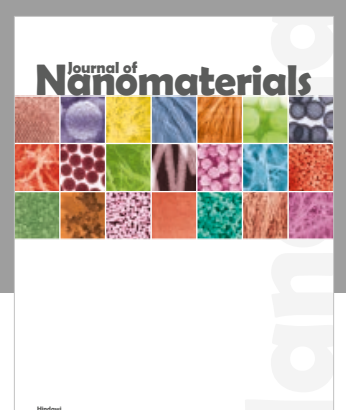

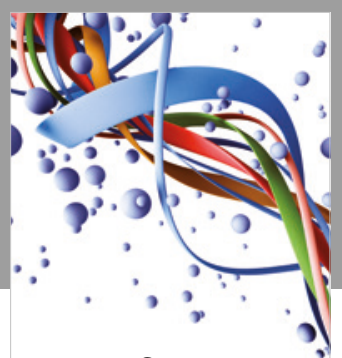

Scientifica

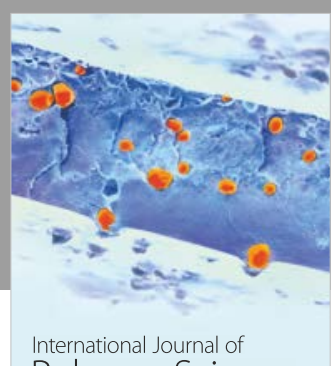

Polymer Science

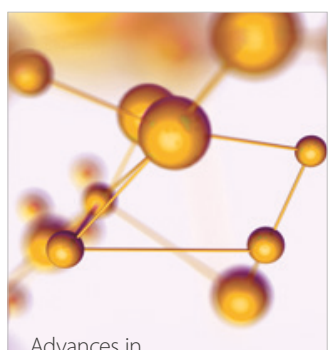

Physical Chemistry
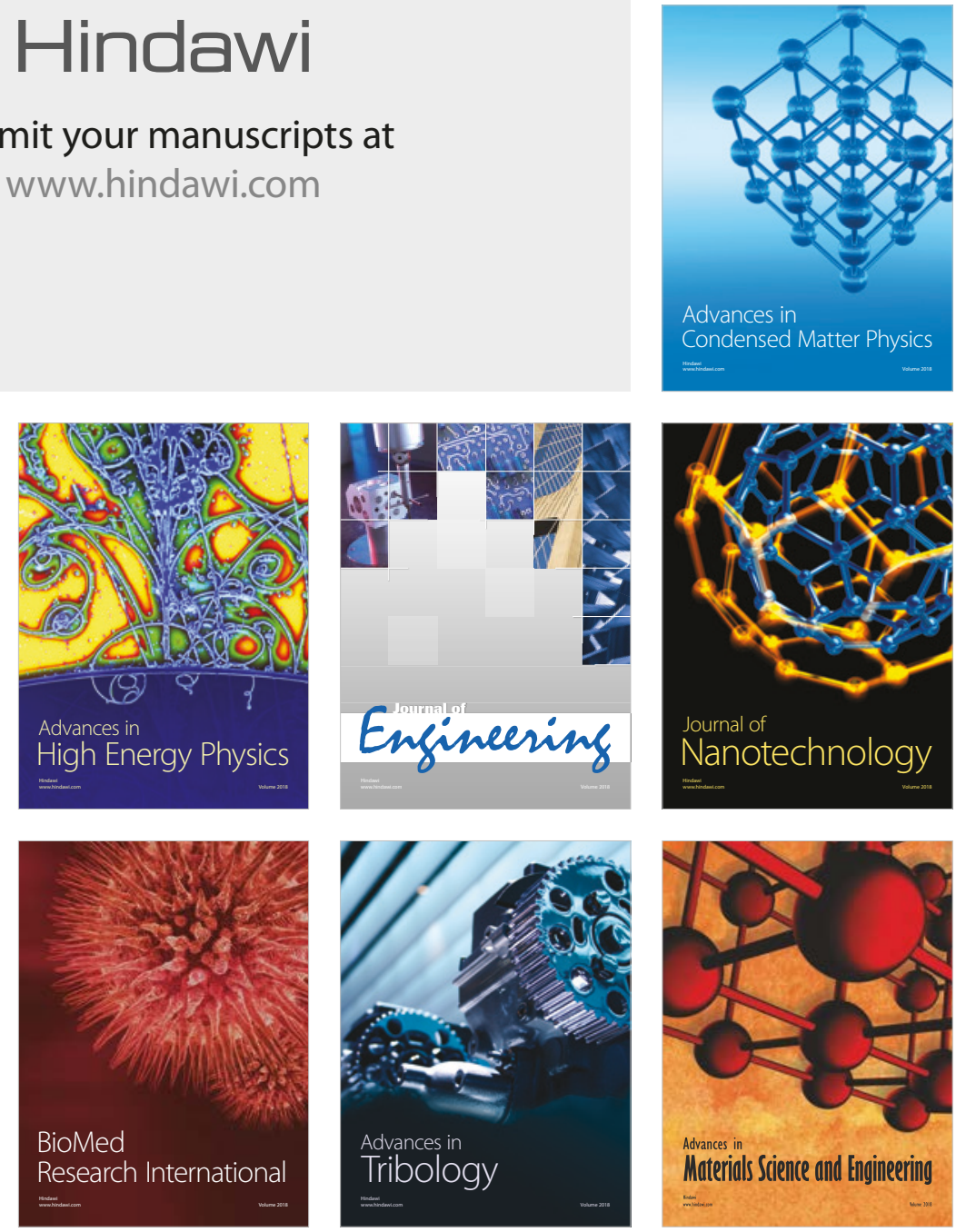